\title{
Benoît Court en sa bibliothèque
}

Quelques indices du travail préparatoire à la rédaction des commentaires aux Arrêts d'Amours de Martial d'Auvergne

\section{Hélène Lannier}

\section{(2) OpenEdition}

1 Journals

Édition électronique

URL : http://journals.openedition.org/aes/1417

DOI : 10.4000/aes. 1417

ISSN : 2258-093X

Éditeur

Laboratoire LISAA

Référence électronique

Hélène Lannier, «Benoît Court en sa bibliothèque », Arts et Savoirs [En ligne], 10 | 2018, mis en ligne le 19 novembre 2018, consulté le 22 avril 2019. URL : http://journals.openedition.org/aes/1417 ; DOI : $10.4000 /$ aes. 1417

Ce document a été généré automatiquement le 22 avril 2019.

Centre de recherche LISAA (Littératures SAvoirs et Arts) 


\title{
Benoît Court en sa bibliothèque
}

\author{
Quelques indices du travail préparatoire à la rédaction des \\ commentaires aux Arrêts d'Amours de Martial d'Auvergne
}

\section{Hélène Lannier}

\section{NOTE DE L'AUTEUR}

L'orthographe française du nom de famille de Benoît Court se trouve sous plusieurs formes dans les sources de seconde main : Court, Lecourt, Le Court ou De Court pour les plus communes. Cette cacophonie des noms a été permise par la rareté et la méconnaissance des sources premières en français qui auraient permis d'attester l'orthographe utilisée à l'époque. Jusqu'à récemment, nous ne connaissions qu'un acte notarié datant de 1554 dans lequel le notaire indique sans ambiguïté que « Fut présent et constituée venerable personne M. Benoist Lecourt docteur es droictz [...]» (ADR 20G11). Nos travaux passés reproduisaient donc cette graphie. Nous changeons ici cette habitude après la découverte d'une deuxième source en français : une annotation manuscrite reportée par Benoît Court à la fin de son exemplaire de la Summa angelica d'Angelo Carletti (1486, Médiathèque de Roanne, Inc 78) dans laquelle il indique se nommer « Benoict Court ». Même s'il n'est pas exclu que l'orthographe de son nom ait varié entre 1518 et 1554, nous nous sommes résolue à adopter cette deuxième orthographe en raison de la nature autographe de l'annotation.

1 En 1533, sort des presses de Sébastien Gryphe à Lyon un ouvrage bien singulier. Intitulé Aresta amorum cum erudita Benedictii Curtii Symphoriani explanatione ${ }^{1}$, le livre reproduit le recueil d'arrêts de Parlement fictifs, les Arrêts d'Amours, composé en français dans les années 1460-1466 par le procureur au Parlement de Paris Martial d'Auvergne et véritable succès de librairie depuis la fin du $\mathrm{Xv}^{\mathrm{e}}$ siècle. Conformément à ce qui est annoncé en page de titre, ces arrêts sont accompagnés des commentaires érudits préparés par le juriste Benoît Court originaire de Saint-Symphorien-le-Château dans les Monts du Lyonnais. Le texte de Martial est continuellement interrompu par les annotations latines de Court qui, prenant pour prétexte des mots ou des sujets évoqués dans les arrêts, entend les 
expliquer par des annotations usant d'une véritable "rhétorique des citations " caractéristique du commentaire juridique de l'époque ${ }^{2}$. Benoît Court fait en effet dialoguer entre elles des références à de très nombreux auteurs et des citations extraites d'innombrables œuvres juridiques comme extra-juridiques. L'index des noms d'auteurs cités dans les commentaires, ajouté aux pièces liminaires de la deuxième édition gryphienne publiée en 1538, témoigne de cette profusion. Comme l'indique son titre, y sont en effet listés les auteurs qui ne sont pas des juristes, au nombre de 246 ; les théologiens, philosophes, historiographes, médecins, orateurs, prosateurs et poètes de langue grecque, latine et vernaculaire ${ }^{3}$ auxquels l'auteur fait référence dans ses commentaires. Le nombre d'auteurs allégués et la diversité des disciplines dont ils relèvent témoignent de la vaste culture encyclopédique de Benoît Court caractéristique de l'idéal du juriste humaniste, de ce jurisconsultus perfectus de la Renaissance décrit par Donald R. Kelley :

In early modern times the jurists'self-image came to resemble Erasmus's man of faith and erudition or even the heaven-storming chameleon featured in Pico's Oration on the Dignity of Man rather than the low-minded mercenary of popular opinion and literary cliché.

Et un peu plus loin :

On the one hand he had to be an encyclopedic scholar, since jurisprudence included (as one sixteenth-century lawyer wrote) "the history of human acts, a knowledge of physics or medecine, mathematics and other matters treated by liberal arts". ${ }^{4}$

Non seulement spécialiste de droit civil et/ou canon, le «juriste parfait » doit également faire preuve de compétences dans toutes les autres sciences et être un fin lettré.

Le nombre pléthorique de sources invoquées par Benoît Court ainsi que la diversité des disciplines et des genres représentés dessinent les contours d'une vaste bibliothèque mentale et posent immédiatement la question de la méthode de travail préalable à la rédaction des commentaires. En l'absence d'un témoignage direct de l'auteur, il est difficile d'y répondre. Nous avons toutefois accès encore aujourd'hui à l'un de ses outils de travail: sa bibliothèque personnelle. Bien qu'actuellement dispersés dans les bibliothèques françaises et étrangères, les livres de Court peuvent en effet être retrouvés grâce au signalement de marques de provenance prouvant leur appartenance à la collection du juriste : reliures aux armes, ex-libris et annotations manuscrites ${ }^{5}$. 
Fig. 1 Reliure aux armes de Benoît Court

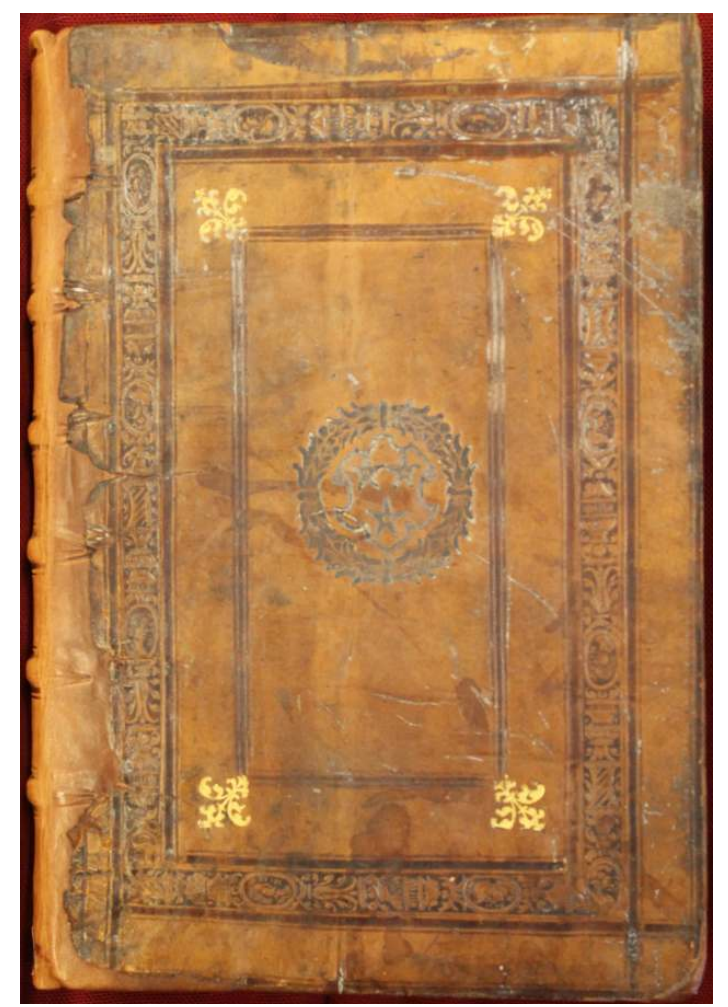

Bibliothèque municipale de Lyon (Rés 334869)

Les livres retrouvés, formant une bibliothèque latine et encyclopédique, complètent alors le portrait d'un Benoît Court en juriste érudit influencé par le mouvement intellectuel et culturel de l'humanisme. L'étude des volumes qui lui ont appartenu permet de confronter la bibliothèque virtuelle mobilisée dans les commentaires et sa bibliothèque réelle, du moins ce que nous en avons retrouvé. Ainsi pouvons-nous, autant que faire se peut, observer le juriste au travail. C'est ce que nous nous proposons de faire ici : rassembler quelques indices présents dans la bibliothèque témoignant du travail préparatoire à l'écriture des commentaires.

\section{Accuratissimi commentarii}

6 L'ouvrage composite rassemblant en recueil les Arrêts d'Amours de Martial d'Auvergne et les commentaires de Benoît Court offre un mélange surprenant de genres et de langues et propose un subtil entremêlement des tons comique et sérieux. Celui-ci est implicitement suggéré dès la page de titre par l'association paradoxale d'un titre d'ouvrage connu comme une fiction comique et parodique et l'annonce de son explication savante. Une attitude visant à jouer avec la matière juridique préside donc à la composition de l'ouvrage et, comme on peut s'y attendre, les commentaires ne sont en réalité pas entièrement sérieux. En effet, de même que Martial d'Auvergne détourne le modèle formel de l'arrêt juridique en le nourrissant d'une matière facétieuse, Court s'est amusé avec la forme et la méthode du commentaire juridique. Il perpétue ainsi une pratique coutumière des praticiens du droit qui, à l'image des clercs de la Basoche parisienne ${ }^{6}$, ont souvent pris la matière juridique comme origine de productions littéraires inventives. 
Alors que, dans son épître dédicatoire, Benoît Court reconnaît avoir pris des risques en publiant une œuvre qui ne fait pas preuve d'une éloquence idéale, il confesse également : «C'est parfois ce que je disais aussi en plaisantant sur l'amour alors que ma plume jouait $\aleph^{7}$. Prenant le risque d'être critiqué aussi pour cela, Court a donc conçu son œuvre comme un divertissement et invite ses lecteurs à jouer avec lui ; il conclut d'ailleurs ses commentaires en déclarant : « Mais déjà, jeunes gens, nous avons suffisamment joué " $^{8}$.

7 Le jeu auquel Court s'adonne dépasse toutefois le simple détournement de la matière juridique pour entrer dans le champ de l'humanisme. D'emblée, la publication des commentaires à Lyon par Sébastien Gryphe, un imprimeur-libraire qui a fait des éditions humanistes de textes antiques sa spécialité ${ }^{9}$, ainsi que le choix de la langue latine et la présence de citations en grec, presque systématiquement accompagnées de leur traduction latine par des humanistes tels qu'Ottmar Nachtgall, Giovanni Battista Pio ou Marsile Ficin, placent l'ouvrage sous la bannière de l'érudition humaniste. De même, les annotations de Court sont caractérisées par le réinvestissement de l'esthétique du serio ludere ou spoudaiogeloion antique qui préside à l'écriture. Comme l'a montré Valérie Hayaert ${ }^{10}$, Court reprend à son compte une posture philosophique symbolisée par les mots d'Horace élevés en maxime : "uertere seria ludo », " mêler le plaisant au sérieux » ( Art poétique, v. 226). Il s'agit donc d'agrémenter les vérités les plus sérieuses de quelques badineries à l'image du "badinage sage ${ }^{11}$ défendu par Érasme dans l'épître dédicatoire de L'Éloge de la Folie:

Chacun peut se délasser librement des divers labeurs de la vie; quelle injustice de refuser ce droit au seul travailleur de l'esprit! Surtout quand les bagatelles mènent au sérieux, surtout quand le lecteur, s'il a un peu de nez, y trouve mieux son compte qu'à mainte dissertation grave et pompeuse. [...] Rien n'est plus sot que de traiter avec sérieux de choses frivoles; mais rien n'est plus spirituel que de faire servir les frivolités aux choses sérieuses. ${ }^{12}$

8 Dans ses commentaires, Court entremêle ainsi des développements savants et sérieux avec des considérations beaucoup plus légères traitant la plupart du temps de l'amour et des mœurs des amants.

L'esthétique sério-comique qui préside à la composition du recueil n'a toutefois pas dispensé le juriste de respecter une méthode de commentaire rigoureuse que les sujets abordés soient sérieux ou non ou qu'un même thème soit traité sérieusement avant de faire l'objet d'une digression facétieuse. Quand il traite de graves questions de droit civil ou canon telles que les héritiers et les témoins, la simonie ou encore les excommunications, ses annotations peuvent être utiles à de jeunes étudiants en droit ou à de savants juristes. Court emploie les abréviations juridiques usuelles pour renvoyer aux textes du droit civil et canon (Digeste, Code, Décrétales de Grégoire Ix, Décrets de Gratien, etc.) et à leurs commentateurs, qu'ils soient civilistes ou canonistes, aux écritures saintes ou aux coutumes. Ces passages de commentaires juridiques conventionnels sont enrichis d'exemples provenant de sources extra-juridiques conformément à un usage qui est attesté depuis le XIV ${ }^{e}$ siècle chez Alberico da Rosate. Ce dernier explique en effet que « Les autorités des poètes et des philosophes peuvent être alléguées dans les causes judiciaires $»^{13}$.

10 Prenons pour exemple un court extrait d'un commentaire à l'arrêt xxIII (P. 230). Alors qu'un amant accuse sa dame de lui avoir versé un seau d'eau froide sur la tête depuis sa fenêtre et de s'être moquée de lui, celle-ci précise que c'est sa chambrière qui est la cause de cette méprise, qu'elle-même ne savait pas que c'était lui qui était en dessous et que, en 
tout état de cause : «Combien quelle luy eust peu mettre remede, car la chose estoit ia faicte ». Court interrompt ici Martial d'Auvergne et, s'appuyant sur les derniers mots du texte «la chose estoit ia faicte », il introduit une règle fondamentale du droit romain connue depuis Aristote et qui consiste à considérer que « ce qui est arrivé ne peut pas ne pas être arrivé $»^{14}$ :

Ce qui est arrivé ne peut pas être annulé par le droit civil (Dig.41.2.1.4.). « Aucune disposition légale ne peut faire que ce qui est fait n'a pas été fait » (Dig.49.15.12.2; § quae igitur. In auth.de aequ.dot.col.vii.). Parce qu'une chose vraie ne doit pas être prise pour de la fiction (Bartole sur le Digeste, § Si vir). En effet, « ce qui est fait est irréversible ", Quintilien (L'Institution oratoire, vii, 4, 4). Plaute dans L'Amphitryon (III, 2) : «Il nie l'évidence ». ${ }^{15}$ De même dans La Marmite ${ }^{16}$, « Que veux-tu ? Le mal est fait. Impossible d'y rien changer » $[. ..] .^{17}$

11 Après avoir cité le Digeste et Bartole, Benoît Court donne des exemples extraits des comédies de Plaute ; sa méthode est donc bien fidèle à celle qu'il annonce dans son épître dédicatoire : «J'ai rassemblé les sentences qui se trouvent tant dans les lois que dans les chapitres de lois et dans toutes autres sources, pour donner un exemple ou une preuve sur un sujet donné » (4).

12 C'est une méthode qu'il emploie aussi dans ses développements plus légers de casuistique amoureuse, par exemple à l'arrêt $\mathrm{xx}^{18}$. Martial d'Auvergne explique qu'un amant a coutume de dormir devant la porte de sa dame mais qu'il réalise un jour que cette dernière n'en sait rien. Il décide alors de lui rendre visite le matin venu. Benoît Court introduit ici un court commentaire :

Devant les portes de leur amante, pour l'apercevoir ou lui parler, les amoureux se promènent, ce dont Tibulle [Élégies, I, 5] ${ }^{19}$ atteste : «Ce n'est pas vain qu'un autre déjà s'arrête sur son seuil, empressé, et souvent guette et bat en retraite, qu'il fait semblant de dépasser la maison, puis bientôt rebrousse chemin dès qu'il est seul et crache cent fois devant la porte. $»^{20}$

13 Les digressions plus légères des commentaires suivent donc la même rigueur méthodologique. Les austères abréviations juridiques renvoyant aux textes du Corpus juris civilis et du Corpus juris canonicus ainsi qu'à leurs commentateurs cohabitent avec les œuvres plus ou moins facétieuses de la littérature antique et moderne et des œuvres d'autres disciplines.

14 Pour répondre à l'exigence herméneutique du commentaire juridique et nourrir le jeu sérieux qui préside à l'écriture de son œuvre, Benoit Court a ainsi brassé des sources nombreuses et relevant de divers genres et disciplines. La lecture des commentaires offre l'image d'une gigantesque bibliothèque mentale et laisse imaginer un travail vertigineux de recherche et de compilation préalable à l'entreprise de rédaction. C'est avec peine que nous imaginons la méthode de collecte et de rédaction suivie par l'auteur. La confrontation de cette bibliothèque imaginaire avec ce que nous connaissons de la bibliothèque réelle permet toutefois de rassembler quelques indices.

\section{La bibliothèque comme outil de travail}

Certaines des mentions, lorsqu'il s'agit d'un titre, d'un nom d'auteur, d'une loi du Digeste ou de courtes citations, ont pu être rapportées de mémoire. L'approximation et les coquilles qui se glissent parfois dans les citations vont dans ce sens. Il est cependant évident que Court n'a pas pu se reposer entièrement sur sa mémoire, il avait donc à sa disposition des notes personnelles ou des livres dans lesquels puiser. Il a pu profiter des 
bibliothèques de ses amis lettrés ou de sa fréquentation des ateliers et des boutiques des imprimeurs-libraires ${ }^{21}$ mais il a également puisé dans sa collection personnelle ou a peutêtre acquis certains ouvrages dans un but utilitaire.

La bibliothèque de Court était semble-t-il importante mais nous ne pouvons en connaître précisément les contours car elle n'est connue ni par un inventaire ni par un catalogue d'époque, seulement d'après les exemplaires qu'il est possible de retrouver dans les bibliothèques publiques et privées lorsqu'ils portent encore les marques de possession du juriste. Notre reconstitution virtuelle de sa collection compte à ce jour un total de 198 titres répartis dans 76 volumes ${ }^{22}$. Si nous nous en référons aux relevés présentés par Pierre Aquilon dans l'Histoire des bibliothèques françaises ${ }^{23}$, il s'agit d'une bibliothèque d'un volume moyen en comparaison des bibliothèques des médecins picards du $\mathrm{XVI}^{\mathrm{e}}$ siècle qui comptent en moyenne cent seize ouvrages et des médecins parisiens du XVII siècle qui cumulent deux cent vingt ouvrages environ. Pierre Aquilon précise que la bibliothèque de l'avocat est « quantitativement voisine de celle du médecin » or, nous savons que Benoît Court est docteur in utroque jure et qu'il a exercé cette fonction dans les années $1530^{24}$ avant d'être nommé chevalier de l'Église de Lyon en $1540^{25}$, ce qui fait de lui également un ecclésiastique ${ }^{26}$.

17 Le contenu de sa bibliothèque est représentatif de la classe d'hommes cultivés, ayant suivi des études et familiers du livre à laquelle il appartient. Ses volumes sont en très grande majorité en latin. Nous avons relevé seulement quatorze titres en français, quatre petites pièces en espagnol et un exemplaire des œuvres de Pétrarque en italien dans l'édition de Jean de Tournes de 1547. Ensuite, dans l'état actuel de la reconstitution, il apparaît que la religion est l'un des centres d'intérêt principaux du juriste. Sans surprise, nous avons recensé deux ouvrages de dévotion : le Breviarium romanum dans sa version réformée par le cardinal espagnol Quiñones ${ }^{27}$ à la demande de Clément VII ainsi qu'un bréviaire manuscrit datant du $\mathrm{Xv}^{\mathrm{e}}$ siècle ${ }^{28}$. La collection recèle des traités théologiques, tels que ceux de Nicolas de Clamanges ${ }^{29}$ et des éditions commentées de textes religieux telle que la traduction d'hébreux en latin de l'Évangile de Saint Matthieu par Sebastian Münster ${ }^{30}$ et les commentaires de l'Épître de Saint Paul aux Romains par Jacques Sadolet ${ }^{31}$ qui se trouvent d'ailleurs rassemblés dans le même recueil factice.

L'Histoire est aussi une discipline très représentée dans la bibliothèque. Nous y trouvons des ouvrages d'histoire religieuse tels que l'Histoires des Hussites de Johannes Colchlaeus ${ }^{32}$ et d'histoire antique (Mœurs des Germains de Tacite dans l'édition établie par Andreas Althamer ${ }^{33}$, l'Ab Urbe condita de Tite-Live $\left.{ }^{34}\right)$. Sont également présents des ouvrages touchant à l'histoire du royaume de France ou des pays limitrophes. Nous avons également recensé de nombreux occasionnels rapportant des faits d'actualité notamment sur les guerres d'Italie ou les guerres contre les Turcs, conservés dans plusieurs recueils factices.

19 D'autres ouvrages appartiennent à la catégorie des Belles Lettres. On trouve notamment un nombre respectable d'œuvres antiques, souvent publiées dans des éditions scientifiques humanistes, parfois accompagnées de commentaires (Aristote, Ovide, AuluGelle, Virgile, Cicéron). Le théâtre est également représenté mais ce sont les recueils poétiques, de poésie le plus souvent morale et religieuse, qui sont les plus nombreux.

20 Le champ des sciences est lui aussi plutôt fourni par des œuvres antiques telles que l' Histoire naturelle de Pline dans une édition commentée par Francesco Massari ${ }^{35}$. On trouve 
également des ouvrages médiévaux ou modernes de mathématiques (Oronce Fine, Charles de Bovelles), des traités de cosmographie et d'astrologie.

21 À regarder l'état actuel du catalogue, nous constatons toutefois des manques. Le droit civil et canon même s'ils sont présents, ne sont pas autant représentés que l'on aurait pu s'y attendre. Dans le domaine canonique, on trouve la Summa Angelica d'Angelus de Clavasio $^{36}$, un glossaire de termes expliqués d'après l'Ancien et le Nouveau Testament ${ }^{37}$ et les commentaires de Jean Quintin sur les Décrétales ${ }^{38}$. Pour ce qui est du droit civil, nous n'avons connaissance que d'un ouvrage: la Forme et ordre de plaidoirie en toutes les cours royalles et subalternes de ce royaume de Jean Bouchet, publiée à Lyon par les frères Frellon en 1543. Il s'agit d'un ouvrage que nous connaissons seulement par une mention de l' Armorial des bibliophiles de Lyonnais, Forez, Beaujolais et Dombes ${ }^{39}$. Les sommes de droit romain et canon citées à presque chaque page du commentaire avec les glosses des juristes médiévaux et modernes devaient certainement se trouver dans la collection. Nous n'en avons toutefois aucune trace. En leur qualité d'usuels, elles n'ont peut-être pas fait l'objet du même soin que d'autres livres considérés comme plus précieux et n'ont ainsi pas reçu de reliure aux armes ni d'ex-libris, ce qui explique peut-être que nous ne les ayons pas retrouvés. Il y a aussi un autre manque : l'exemplaire des Arrêts d'Amours de Martial d'Auvergne à partir duquel le juriste a travaillé pour préparer ses commentaires. En 1827, Nicolas-François Cochard signalait posséder un manuscrit autographe des Aresta amorum mais nous ne savons si ce document a réellement existét ${ }^{40}$.

22 Afin de mieux appréhender le travail mené par Court pour la rédaction de ses commentaires aux Arrêts d'Amours, nous voudrions croiser le contenu de la bibliothèque mentale qui s'en dégage avec le contenu de la bibliothèque réelle de Court. Cela ne peut se faire qu'en retenant les ouvrages dont la date de publication est antérieure à celle des Aresta amorum, soit 1533. Et encore, les titres restants sont à prendre avec précaution puisque, à de très rares exceptions près, nous ne pouvons savoir à quel moment un ouvrage a réellement fait son entrée dans la collection. Nous avons relevé 95 éditions publiées avant 1533, ce qui représente environ $48 \%$ de la collection. À cela s'ajoutent trois ouvrages publiés en 1533 que Court a pu avoir entre les mains juste avant la publication de son œuvre. Dans la catégorie des incertains s'ajoutent treize titres qui sont des plaquettes d'occasionnels ou de pièces poétiques dont les dates de publication ne sont pas connues. Toujours est-il qu'environ la moitié de la bibliothèque a pu être utilisée par le juriste pendant la préparation de ses commentaires. Malgré cet ensemble relativement important, il est difficile d'établir des correspondances attestées entre ouvrages cités et ouvrages possédés, les liens sont ténus pour les raisons que nous venons d'évoquer mais aussi parce que finalement peu d'auteurs et d'œuvres cités dans les commentaires se trouvent dans la bibliothèque. Nous nous proposons de présenter tout de même ici les cas que nous avons pu relever.

\section{Titres cités et titres possédés}

23 Certains des livres qui se trouvent dans la bibliothèque réelle de Benoit Court ont pu l'aider à rédiger ses commentaires. Les recherches pour la rédaction des passages mentionnant des autorités religieuses ont pu, par exemple, être nourris des lectures du Catalogus scriptorum ecclesiasticorum de Jean Trithemius dont la date de publication est antérieure à celle des arrêts ${ }^{41}$. 

éditions antiques ayant pu aider à la préparation des commentaires. Il est toutefois surprenant de constater que la date d'édition de seulement deux ouvrages de la bibliothèque est antérieure à la publication des Aresta amorum. Il s'agit d'un manuscrit de la Vie des douze Césars de Suétone qui, d'après la British Library, daterait de la fin du XV siècle ${ }^{43}$. Cette œuvre est mentionnée aux pages 120 et 285 du commentaire, c'est donc peut-être sur ce manuscrit que Benoît Court s'est appuyé. Le deuxième ouvrage est l'édition princeps d'un recueil de Stéphano Negri (1517) renfermant une traduction latine de l'Heroicus attribué à Philostrate l'Athénien et d'un dialogue paraphrasant la Description de la Grèce de Pausanias ${ }^{44}$. Ce volume a probablement servi au travail préparatoire de Court sur ses commentaires, quelques annotations marginales de sa main se trouvent d'ailleurs à l'intérieur. Stefano Negri est mentionné à deux reprises dans les commentaires (p. 240 et 310 ) mais Court renvoie respectivement à la troisième et à la première de ses Chriae quinque, des œuvres qui ne se trouvent pas dans le volume de la bibliothèque. En revanche, Philostrate et son Heroicus apparaissent aux pages 312 et 263 des Aresta amorum. À cette dernière page en particulier, nous les retrouvons dans une glose sur l'assassinat par empoisonnement où il est question de Circé. Court indique que Philostrate appelle cette dernière « dea venenorum », terme que l'on retrouve bien au f. 16 r. dans la traduction de Negri. Quant à la Description de la Grèce de Pausanias et plus précisément au livre viı portant sur l'Achaë, ils sont mentionnés à la page 29. Court relate le mythe du berger Sélemnos changé en fleuve par Vénus après que la nymphe Argyra s'est lassée de lui ${ }^{45}$. Si cet épisode du texte de Pausanias est bien présent dans le dialogue de Negri au f. 186 v., il n'y a pas de réelle correspondance entre le texte de Negri et de Court. D'autant que ce dernier donne une citation exacte du texte grec $^{46}$ et sa traduction latine dont l'origine n'est pas précisée. Court a donc eu recours à une autre édition en grec et à sa traduction latine. À moins que cette dernière ne soit l'œuvre du juriste luimême dont il est probable qu'il ait été hellénisant. Pour aller dans ce sens, en plus des citations grecques qui émaillent ses commentaires, nous pouvons signaler que sa bibliothèque contient les Institutiones graecae grammatices d'Urbano Valeriani ${ }^{47}$. En dehors des deux volumes que nous venons de mentionner, nous n'avons à ce jour pas retrouvé les éditions des œuvres antiques que Court a pu utiliser pour préparer ses commentaires bien que ceux-ci regorgent de références aux orateurs, philosophes, poètes, dramaturges et historiens de l'Antiquité grecque et romaine. Les éditions classiques qui se trouvent dans notre reconstitution de la bibliothèque datent principalement des années 1530-1540. Nous pouvons donc supposer qu'un pan important de la bibliothèque n'a pas encore été retrouvé ou bien que Benoît Court a alors puisé dans les collections de ses amis.

Les commentaires aux Arrêts d'Amours présentent des vers de nombreux poètes italiens modernes, un intérêt qui se lit aussi dans la bibliothèque. Elle renferme en effet plusieurs recueils poétiques tels qu'un recueil factice conservé à la Bibliothèque nationale de 
France qui rassemble l'Anno mundi 5270 de Bernardino Boccacio, les épigrammes sacrées et satiriques de Luigi Bigi Pittorio, le recueil de distiques de Benedetto Giovio, le Dialogus tripartitus et les épigrammes Noël Chamaillard, le De laudibus deiparae virginis opusculum de Franciscus Belossus et, enfin, la De Francquillongo canore Ode super saluberrimo humane redemptionis mysterio de Johannes von Franken ${ }^{48}$. Parmi ces auteurs, Boccacio, Pittorio et Giovio sont mentionnés dans les commentaires respectivement aux pages 347, 259 et 338. Pour le premier, il s'agit d'une simple mention de son nom sans citation. En revanche, pour les deux derniers, l'un de leurs poèmes est cité. Ainsi, l'épigramme Ad Ursum de Pittorio est reproduite à la page 259 , or c'est un texte que l'on trouve dans le recueil possédé par Court. Dans ce même exemplaire, se trouvent aussi quelques épigrammes de Fauste Andrelin dont un distique satirique sur les médecins que Court mentionne dans une annotation contre les médecins à la page 223 des commentaires. Enfin, le distique de Benedetto Giovio qui se trouve à la page 338 est bien présent dans l'édition de ses poèmes possédée par le juriste.

Ensuite, un recueil factice conservé à la bibliothèque de l'Arsenal et rassemblant plusieurs œuvres de Nicolas Barthélemy de Loches, moine bénédictin et professeur de droit, présente également l'intérêt de renfermer une édition de sa tragédie religieuse Christus Xylonicus. Court pouvait avoir ce volume entre les mains au moment d'en citer un court passage à la page 188 des Aresta amorum ${ }^{49}$. Dans une courte annotation visant à démontrer que l'ivresse révèle ce que l'on cache («Ebrius revelat arcana »), le juriste extrait quelques mots de la tirade d'un des personnages: "Arcana vinum detegit » (II, 4, f. $\mathrm{c}^{2}$ ). Une autre pièce de théâtre qui se trouve dans la bibliothèque est également mentionnée dans les commentaires. Il s'agit de la comédie humaniste Dolotechne composée à fin $\mathrm{du} \mathrm{xv}^{\mathrm{e}}$ siècle par le vénitien Bartholomeo Zamberti. Benoît Court en cite quelques vers aux pages 241 et 404 de ses commentaires. La première citation est insérée dans une courte annotation aux côtés d'une tirade extraite de la Casina de Plaute pour expliquer que l'amour rend coquet («Amoris nitidus »). Quant à la deuxième évocation de la pièce, elle intervient à la toute fin des commentaires dans une annotation visant à démontrer que le mieux est de ne pas se quereller pour éviter les procès ("Non litigandum »). C'est probablement dans l'édition princeps qui se trouve dans sa collection que Court a puisé ces extraits ${ }^{50}$.

Les auteurs et les titres que nous venons de citer, parce qu'ils se trouvent à la fois dans les commentaires et dans la bibliothèque personnelle de Benoît Court, ont pu être des outils utilisés par le juriste pour élaborer son œuvre. Nous n'avons toutefois aucune preuve incontestable du réel emploi qu'il en a fait. Deux autres volumes présentent quant à eux des traces de lecture qui sont des preuves plus tangibles de l'utilisation des livres.

\section{Marginalia et marques de lecture}

Benoît Court semble avoir rarement laissé d'annotations manuscrites dans ses livres, les traces de sa main éclairant la genèse des commentaires sont donc très rares. Son écriture est toutefois repérable par endroits. Nous pouvons l'authentifier grâce à un manuscrit conservé à la Bibliothèque municipale de Lyon qui ne laisse aucun doute quant à son caractère autographe. Il s'agit de notes rassemblant les biographies des papes jusqu'à la fin du $\mathrm{IX}^{\mathrm{e}}$ siècle ${ }^{51}$. Court le commence en précisant: "Haec a me Benedicto Curtio Symphoriano equite ecclesiae Lugdunensis transcripta sunt ex antiquissimo libro [...] $\aleph^{52}$. 
Fig. 2. Manuscrit autographe

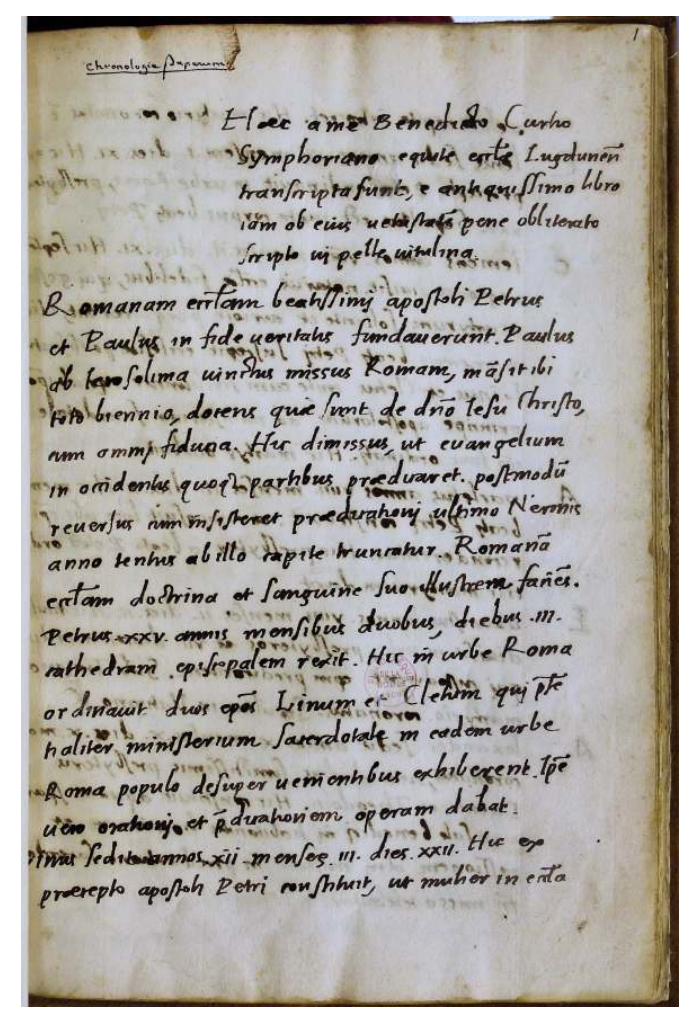

Bibliothèque municipale de Lyon (Rés. Ms 195 T. 02)

Ce témoignage facilite l'identification de la main du juriste dans les marges de ses livres et évite de la confondre avec la main de son frère, Léonard, qui a abondamment annoté quelques livres de la bibliothèque.

31 C'est le cas d'une édition rassemblant plusieurs œuvres de l'écrivain et humaniste Platina 53. Court a annoté le premier ouvrage du recueil, le Liber de vita Christi ac omnium pontificum, ainsi que le dialogue Contra amores. Ce dernier est très succinctement mentionné à la page 338 des commentaires, à la toute fin d'une annotation sur les peines d'amour: "Barptolomeus Platina ad Ludouicum Stellam dialogum in amores scripsit», i.e. «Bartholomaeus Platina a écrit un dialogue contre les amours à l'intention de Ludovicus Stella ». Composé vers 1467-68, le Contra amores s'inscrit dans la tradition antiérotique présente en Italie dès la première moitié $\mathrm{du} \mathrm{XVI}^{\mathrm{e}}$ siècle ${ }^{54}$. L'auteur se met en scène dans un dialogue avec Stella, un jeune homme en proie à la passion amoureuse et développe une satire de l'amour charnel pour tenter de convaincre son ami des dangers qu'il encourt et de le persuader de rejeter la passion érotique.

En raison du thème que ce court dialogue développe, il est plus que probable que Court s'en soit inspiré pour développer ses propres passages satiriques sur l'amour. Les quelques annotations marginales qui parcourent son exemplaire vont dans ce sens. 
Fig. 3 Première page du Dialogus contra amores de Platina

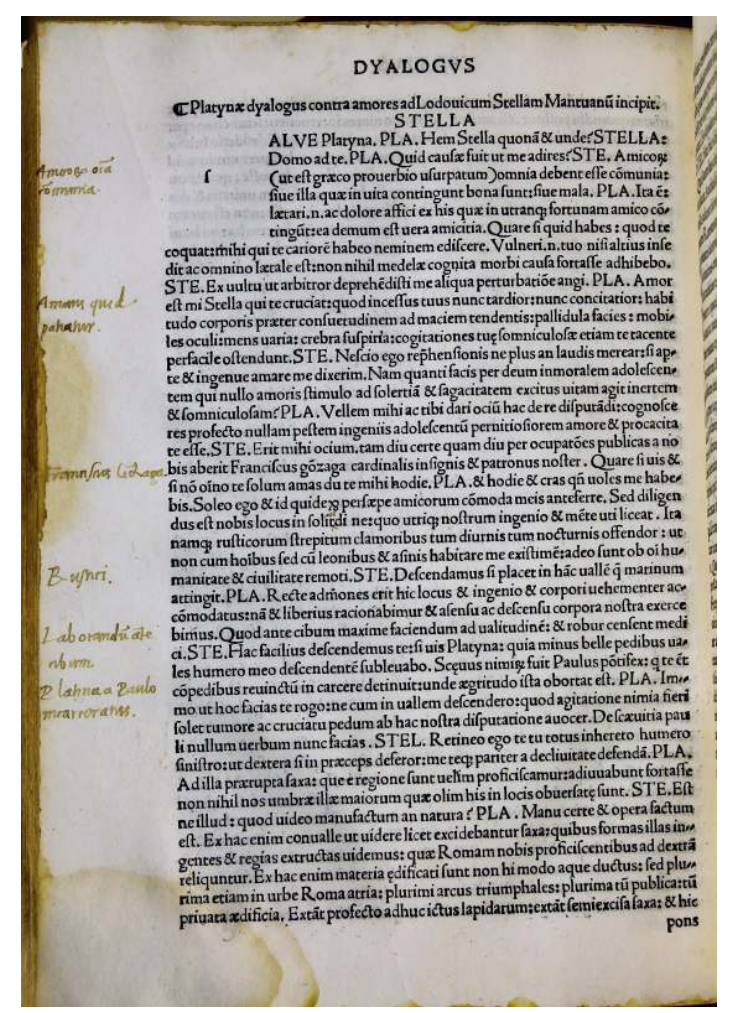

f. $C^{1}$ V., Bibliothèque municipale de Lyon (Rés. Ms 195, T. 01)

Par exemple, la note " amorus quid patiat », « ce que l'amour fait endurer » que l'on peut voir sur la reproduction (fig. 3), en marge de la première page du recueil ${ }^{55}$ est à mettre en parallèle des nombreuses annotations décrivant les effets du sentiment amoureux que l'on trouve dans les commentaires. Parmi les symptômes physiques, Platina mentionne la "pallidula facies", i.e. "figure pâlotte" qui rappelle par exemple l'annotation dans laquelle Court développe l'idée que l'« amor languentes et pallidos facit ", «l'amour rend indolent et pâle ». (p. 266)

Les livres de Court présentent d'autres marques de lecture beaucoup plus difficiles à attribuer tels que des soulignements, des accolades ou des manicules. Un volume conservé à la bibliothèque municipale de Lyon présente toutefois deux " surlignements » forts intéressants. Il s'agit de la correspondance de Saint Sidoine Apollinaire dans une édition établie et commentée par Giovanni Battista $\mathrm{Pio}^{56}$ et il se trouve que deux extraits de celle-ci se lisent dans les commentaires. D'abord à la page 58, alors que Court prétend démontrer que «similes mores conciliant amorem », i.e. "Des mœurs similaires facilitent l'amour ", il introduit la citation exacte qui est comme « surlignée » dans le volume de la bibliothèque (fig. 4$)^{57}$. 
Fig. 4. Correspondance de Saint Sidoine Apollinaire

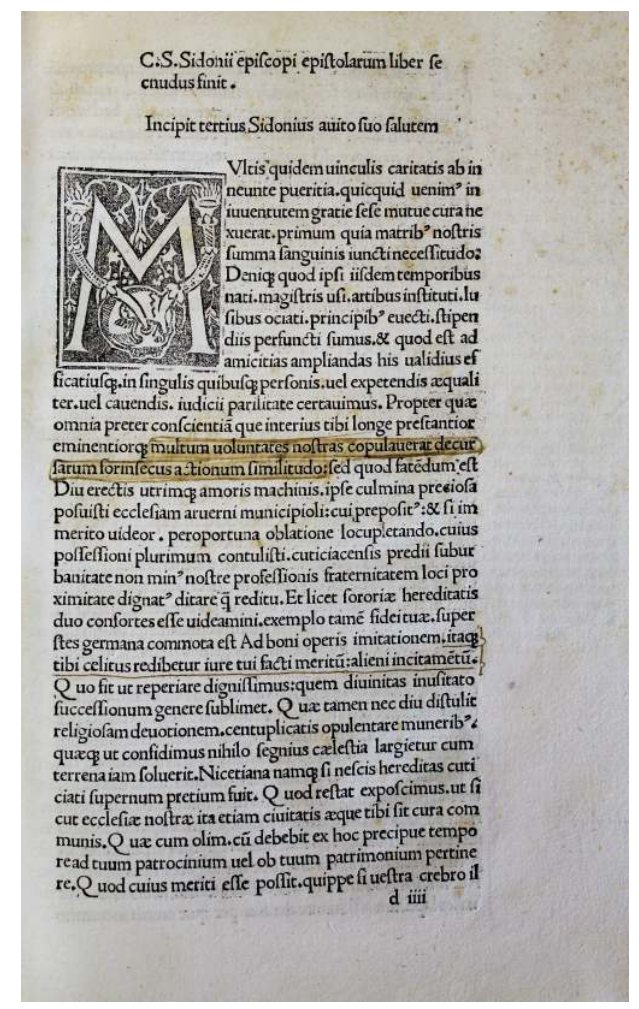

Bibliothèque municipale de Lyon (Rés Inc 158 (1), f. $d^{4}$.

Puis, au passage de la page 288 à la page 289, dans une annotation expliquant qu'il faut se fier aux experts qui interviennent dans le cadre des procès, le juriste insère la citation exacte surlignée dans le volume (fig. 5) ${ }^{58}$. 


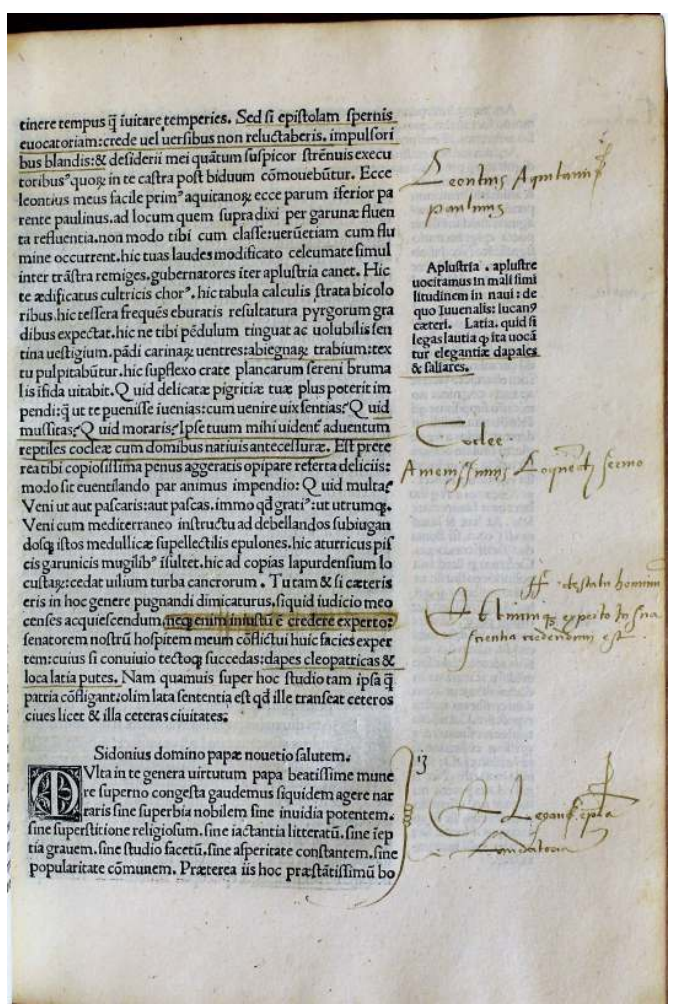

Bibliothèque municipale de Lyon (Rés Inc 158 (1), f. k V.

On remarque de plus, en regard du passage, une annotation marginale renvoyant au titre du Digeste intitulé «De statu hominum» (Dig. 1.5) ainsi que la maxime médiévale : «Et unicuique experto in sua scientia credendum est ", i.e. "Chaque expert dans son domaine doit être cru ». Ces annotations qui sont de la main d'un juriste auraient pu être l'œuvre de Court et se retrouver dans les commentaires. Toutefois, il n'en est rien. Il semble que cette main soit davantage celle de Léonard Court qui a également laissé sa marque dans ce volume par sa signature et de nombreuses annotations. Comme Benoît, Léonard, qui était notaire, devait lui aussi maîtriser les références juridiques.

Les correspondances entre les sources convoquées par Benoît Court dans ses commentaires aux Arrêts d'Amours et les ouvrages qu'il a effectivement possédés sont finalement peu nombreuses au regard des chiffres qui pouvaient laisser supposer beaucoup plus de possibilités. Celles que nous avons pu rassembler donnent tout de même quelques indices sur la genèse des annotations depuis les simples titres à la fois cités et possédés jusqu'aux témoignages d'une lecture savante au cœur même des livres. La bibliothèque réelle du juriste, en l'état actuel de notre reconstitution, est donc loin de recouvrir la bibliothèque mentale que nous percevons au travers des commentaires. Il semble toutefois probable que les livres mentionnés plus haut aient été des outils utilisés lors du travail de compilation préalable à l'écriture, que ce soit pour les développements juridiques ou pour les développements plus légers traitant du sentiment amoureux. Quoi qu'il en soit, la collection personnelle de Benoît Court, telle qu'elle nous apparaît au travers des titres qu'il a été possible de rassembler, prend l'apparence d'une bibliothèque encyclopédique, représentative des cercles cultivés auxquels le juriste appartient et qui témoigne d'une vaste culture dépassant les strictes connaissances nécessaires à l'exercice 
de ses fonctions. Les matières représentées y sont aussi diverses que celles que nous retrouvons dans les commentaires. Aussi complète-t-elle le portrait de Court en un jurisconsultus perfectus faisant preuve de la curiosité intellectuelle et de la culture encyclopédique qui se manifeste également dans son œuvre.

\section{NOTES}

1. Aresta amorum. Cum erudita Benedicti Curtii Symphoriani explanatione. Lugduni apud Seb. Gryphium, 1533, 4, [1-1bl-5-1bl-], 321 [i.e. 323-2-3bl], [5] p. Dans la suite de cet article, les références à l'œuvre de Benoît Court seront données d'après Snotre édition de référence, la troisième et dernière édition gryphienne de cet ouvrage, publiée en 1546: Aresta amorum LI. Accuratissimis Benedicti Curtii Symphoriani commentariis ad utriusq[ue] iuris rationem forensiumque actionum usum, quam acutissimè accommodata. [...] Apud Seb. Gryphium, Lugduni, 1546, 8 , 430, [18] p.

2. Marc Fumaroli, L'Âge de l'éloquence. Rhétorique et "res literaria» de la Renaissance au seuil de l'époque classique, Paris, Albin Michel (« Bibl. de l'Évolution de l'Humanité »), 1994, p. 444.

3. Le titre de l'index indique exactement : «Autorum nomina, quorum testimonio autor usus est in his scholiis : eo uidelicet ordine, ut primum Grceci, post Latini: dein vulgares, et inter hos omnes primum Theologi, post: Philosophi, Medici, Historiographi, Oratores, et omnes qui oratione soluta scripserunt: postremum Poētce locati sunt. », p. 8-10.

4. Donald R. Kelley, "Jurisconsultus Perfectus: the lawyer as Renaissance man", Journal of the Warburg and Courtauld Institutes, 51 (1988), p. 85 et p. 89 citant Antoine Favre, De jurisprudentiae papinianae scientia ad ordinem institutionum imperialium efformata [...], Lugduni, Apud horedes Iacobi Chouët, MDCVII, [préface au lecteur].

5. Pour plus de précisions sur les marques de provenance des livres de Benoît Court, voir «Reconstituer une bibliothèque $\mathrm{du} \mathrm{XVI}{ }^{\mathrm{e}}$ siècle : la bibliothèque de Benoît Lecourt " [en ligne], article mis en ligne le 12/11/2015 sur le site de l'association de doctorants les Têtes Chercheuses, <teteschercheuses.hypothèses.org>. Voir aussi Julien Baudrier, Léon Galle, William Poidebard, Armorial des bibliophiles de Lyonnais, Forez, Beaujolais et Dombes, Lyon, Société des bibliophiles lyonnais, 1907, p. 347-349 ; Nicolas-François Cochard, St-Symphorien-sur-Coise et ses environs, Paris, Res Universis, 1989, p. 129-134, fac-similé de l'édition de 1827 parue sous le titre Notice historique et statistique du canton de Saint-Symphorien-le-Château; Guy Parguez, "À propos de Benoît Lecourt ", Saint-Symphorien-sur-Coise et sa région, Lyon, Union des sociétés historiques du Rhône, 1999, p. 21-31.

6. Voir à ce sujet Marie Bouhaïk-Gironès, Les clercs de la Basoche et le théâtre comique (Paris, 1420-1550), Paris, H. Champion, 2007.

7. « Nonnumquam etiam quod in amore iocatus sim lasciuiente calamo", p. 4.

8. «Sed iam satis iuuenes lusimus, [...] », ibid., p. 404.

9. Sur Sébastien Gryphe, voir Raphaële Mouren (dir.), Quid novi? Sébastien Gryphe à l'occasion du $450^{e}$ anniversaire de sa mort. Actes du colloque du 23 au 25 novembre 2006, Villeurbanne, Presses de l'Enssib, 2008.

10. Valérie Hayaert, "Serio ludere et humanisme juridique : les gloses de Benoît Le Court aux Arrêts d'Amour de Martial d'Auvergne " dans Géraldine Cazals et Stéphan Geonget (dir.), Des "Arrests Parlans ». Les Arrêts notables à la Renaissance entre droit et littérature, Genève, Droz, 2014, p. 107-108. 
11. Patricia Eichel-Lojkine, Excentricité et humanisme. Parodie, dérision et détournement des codes à la Renaissance, Genève, Droz, 2002, p. 92.

12. Érasme, Éloge de la folie, Pierre de Nolhac (trad.), Paris, Garnier-Flammarion, 1964, p. 14. Texte latin d'Érasme, Éloge de la folie, Pierre de Nolhac (éd. et trad.), Paris, Garnier, 1953 («Classiques Garnier »), p. 4-5 : «Nam quae tandem est iniquitas, cum omni vitae instituto suos lusus concedamus, studiis nullum omnino lusum permittere, maxime si nugae seria ducant, atque ita tractentur ludicra, ut ex his aliquanto plus frugis referat lector non omnino naris obesae, quam ex quorundam tetricis ac splendidis argumentis? [...] Ut enim nihil nugacius, quam seria nugatorie tractare, ita nihil festivius, quam ita tractare nugas, ut nihil minus quam nugatus fuisse videaris ».

13. Traduction de Valérie Hayaert, Mens emblematica et humanisme juridique. Le cas du Pegma cum narrationibus philosophicis de Pierre Coustau (1555), Genève, Droz, 2008, p. 5, d'après Ernst H. Kantorovicz, "The Sovereignty of the Artist : A note on Legal Maxims and Renaissance Theories of Art " dans De artibus opuscula XL, Essays in honor of Erwin Panofsky, Milliard Meiss (éd.), New York, New York University, 1961, vol. 1, p. 267-279. Texte original : «Authoritates poetarum et philosophorum [...] possint in causis allegari », In primam [-secundam] codicis partem commentarii [...], Venezia, ad signum Aquilae renovantis, 1585-1586, f. 63 et au f. 3r : " Allegat etiam haec scientia poetas ", "Cette science [la science juridique] allègue aussi les poètes ».

14. "Factum infectum infieri non potest ", voir Jean Névizan, Syluae nuptialis libri sex [...] Ioanne Neuiziano Astensi, Iurisconsulto clarissimo, authore [...], Lugduni, sub scuto coloniensi, [A. Vincent], 1545 [éd. princeps Paris, P. Vidoue pour J. Kerver, 1521], p. 29 (24a). Sur cette règle juridique, voir Stephan Geonget, La notion de perplexité à la Renaissance, Genève, Droz, 2006, p. 115.

15. Plaute, Comédies, Alfred Ernout (trad.), Paris, Les Belles Lettres, 1989, t. I, p. 59. Littéralement : « Il déclare que ce qui a été fait, n'est pas fait. »

16. Ibid., p. 192.

17. « Factae enim res infirmari iure ciuili non possunt.l.j.§.si uir.ff.de acqui.poss. Factae aute[m] causae infectae nulla constitutione fieri possunt.l.in bello.§.factae.ff.de rap.§quae igitur.in auten.de aeq.dot.col.vij. Quod re uera, non fictione intelligendum est. Bart. In d.\$.si uir. Immutabile enim est quod factum est. Quintilianus lib. Vij.c.v.Plautus in Amphytrione: / Ea quae sunt facta, infecta esse acclamitat. I Idem Aulularia. I Qua uis fieri? factum est illud fieri infectum non potest. I Agatho apud Aristotelem lib.vj.c.ij.Ethico. [...]», p. 230.

18. Ibid., p. 195.

19. Traduction française de Tibulle et les auteurs du corpus tibullianum, Max Ponchont (éd.), Paris, Les Belles Lettres, 1961, p. 41.

20. "Ante fores amasiae, ut eam aspicere possint, aut alloqui, deambulant amatores, quod testatur Tibullus : | Non frustra quidam iam nunc in limine perstat / Sedulus, ac crebro prospicit, ac refugit: | Atque simul transire domum, mox deinde recurrit / Solus, et ante ipsas excreat usque fores. », p. 195.

21. Voir notre article «Benoît Lecourt dans l'atelier de Sébastien Gryphe (1533-1546) », Gryphe, Revue de la Bibliothèque municipale de Lyon, $\mathrm{n}^{\circ}$ 26, novembre 2016, p. 28-33.

22. La collection de Court comprend un nombre important de recueils factices, ce qui explique l'importante différence entre le nombre de titres et le nombre de volumes.

23. Pierre Aquilon, «Petites et moyennes bibliothèques (1530-1660)» dans Claude Jolly (dir.), Histoire des bibliothèques françaises. Les bibliothèques sous l'Ancien Régime (1530-1789), [Paris], Promodis - Éditions du Cercle de la Librairie, 1988, p. 183-184.

24. Dans son recueil de 1537, le poète Claude Rousselet adresse une épigramme à « Aduocato uitae inculpatae Benedicto Curtio", i.e. : «À Benoît Lecourt, avocat à la vie irréprochable ». Claude Rousselet, Claudii Rosseletti jureconsulti, patritiique Lugdunensis epigrammata, Lyon, Sébastien Gryphe, 1537, p. 46.

25. Au nombre de sept, les chevaliers de l'Église étaient les juristes chargés de défendre les intérêts temporels de l'Église de Lyon. 
26. Il aurait bénéficié de la cure de Coise, dans les Monts du Lyonnais, et pour être nommé chevalier, il fallait être au moins sous-diacre et licencié en droit. Voir Jean Beyssac, Les chevaliers de l'Église de Lyon, Lyon, Imprimerie des missions africaines, 1925, p. 91.

27. Breviarum Romanorum ex sacra potissimum scriptura, scripture et probatis sanctorum historiis nuper confectum [...], Lugduni, (Excudebat Baltazard Arnoullet, una cum haeredibus Ioannis Barbous), $1544,4^{\circ}$, [54], 255 f. Bibliothèque municipale de Lyon (BmL) (Rés 105557).

28. [Bréviaire], s.l., [XIV siècle], manuscrit sur parchemin, Bibliothèque publique d'Evora (CXXIV/2-21).

29. Nicolai de Clamengiis, de lapsu et reparatione justiciae libellus. Eiusdem disputatio super materia concilii generalis. Item Libellus apostolorum nationis Gallicanae, [...], [Bâle], [A. Cratender], [ca. 1520], $4^{\circ}$, [6] 130 [2] p., BmL (Rés 334869).

30. Euangelium secundum Matthaeum in lingua Hebraica, cum uersione Latina atque succinctis annotationibus Sebastiani Munsteri, Basileae (apud Henricum Petrum), mense martio 1537, $2^{\circ}$, [8], 154, [2] p. BmL (Rés 100090).

31. Jacobi Sadoleti [...] In Pauli Epistolam ad Romanos commentariorum libri tres, Lugduni apud Sebastianum Gryphium, 1535, 2², 230, [4] p. BmL (Rés 100091).

32. Historiae Hussitarum libri duodecim per Ioannem Cochlaeum, apud S. Victorem prope Moguntiam, ex officina Francisci Behem Typographi, 1549, 2 , [1-1bl-30] 599 [-1] p. BmL (Rés 105165).

33. Commentaria Germaniae in P. Cornelii Taciti equitis Rom. libellum de situ, moribus, et populis Germanorum [...] Andreae Althameri diligentia, pro suo erga Germaniam amore, elucubrata, Norimbergae apud Ioh. Petreium, anno 1536, 4 , [48], 341, [3] p. BmL (Rés 317087).

34. Tite-Live, Historiae principis. Decas prima, Lyon, Sébastien Gryphe, $1548,16^{\circ}$, ouvrage $n^{\circ} 163$ signalé dans le catalogue de Wemaëre-de Beaupuis-Denesle-Binoche et Giquello pour la vente d'ouvrages provenant du château de Beaumesnil (Fondation Fürstenberg-Beaumesnil), le 9 décembre 2013.

35. Francisci Massarii Veneti in nonum Plinii De naturali historia librum Castigationes et Annotationes [... ], Basileae, (apud Hieronymum Frobenium \& Nicolaum Episcopium), Anno 1537, $4^{\circ},[16] 367$ [16] p., BmL (Rés 357291).

36. [Summa angelica de casibus conscientiae], [s.l.s.n.], (1486), 387 f. Médiathèque de Roanne (Inc 78).

37. Incipiunt exempla sacre scripture ex utroque testamento $s c d m$ ordinem litterarum collecta et primo de Abstinentia, [Paris], [Ulrich Gering, Martin Cranz, Michael Friburger], [ca. 1473], [101] f., Österreichische nationalbibliothek Wien (Ink.2.G.21.).

38. [Exegesis concilij cuiusdam generalis, in vno beneficiorum multitudinem, vetantis tertio libro Decretalium Gregorianarum, [...] ex commentariis rerum quotidianarum Io. [...]], [Parisiis], [ex officina Christiani Wecheli], [1539], $4^{\circ}$, [180] f. (page de titre manquante), BmL (Rés 334870).

39. Julien Baudrier, Léon Galle, William Poidebard, Armorial des bibliophiles de Lyonnais, Forez, Beaujolais et Dombes, Lyon, Société des bibliophiles lyonnais, 1907, p. 349. Nous ne connaissons pas le lieu de conservation actuel de cet ouvrage. Au moment de la publication de l'Armorial, il appartenait à la bibliothèque privée de Julien Baudrier.

40. Nicolas-François Cochard, St-Symphorien-sur-Coise et ses environs, Paris, Res Universis, 1989, p. 131-132, fac-similé de l'édition de 1827.

41. Catalogus scriptorum ecclesiasticorum, siue illustrium virorum [...]. Per venerabilem virum, Dominum Iohannem à Tritenhem [...], [Cologne], [Pierre Quentel], Anno 1531, 4 , [4] CLXXXIIII f., BmL (Rés 357319).

42. Militia Francorum regum pro re christiana, [...] [Paris], Vaenundatur cum quibuldam Ioannis Pyrrhi pigram matis in aedibus Afcenfianis, [1518], $8^{\circ}$, [12] f., Bibliothèque Méjanes (Rés. D. 0487).

43. Gaius Suetonis tranquilli de vita duodecim caesarum, [Italie, fin du $\mathrm{xv}^{\mathrm{e}}$ siècle], [175] f., British Library (Add MS 57911). 
44. Stephani Nigri [...] Dialogus, quo quicquid in graecarum literarum penetralibus reconditum [...] in lucem pro pagatur. His accedunt Philostrati Heroica [...], ([...] Mediolani, Alessandro Minuziano, 1507), $2^{\circ}$, [18] 211 [i.e. 210] [13] f.

45. Voir Pausanias, Description de la Grèce, VII, 23, 1 à 3.

46. Ibid., VII, 23, 3.

47. Institutiones graecae grammatices, (Venetiis in aedibus Aldi Manutii Romani, 1497), $4^{\circ}, 214 \mathrm{f}$., collection privée.

48. Anno mundi. 5270. B. Boccaccius, (Impressum Papiae), 1514, (per Magistrum Bernardinum de Haraldis), $8^{\circ}$, [8] 47 [1 blc.] f. ; Pictorii Sacra et Satyrica Epigrammata [...], [s.l.n.d.], [20] f. ; Benedicti Iouii Nouocomensis Disticha [...], [12] f.; Dialogue tripertitus [...] cum nonnullis Epigrammatibus a Petro Chamaillard [...] editus, [Paris], [Gilles et Jean I de Gourmont], [s.d.], 8, [8] f.; Francisci Belossi forcalqueriensis de laudibus Deiparae Virginis opusculum per quam lepidum, Impressum Auinione per [...] Johannem de Channey impreflorem, [s.d.], $8^{\circ}$, [20] f.; De Franquillongo canore ode super saluberrimo humane redemptionis mysterio, [Paris], [Hémon Lefèvre], [ca. 1520], $8^{\circ}$, Bibliothèque nationale de France (RES P- YC- 63).

49. Nicolai Barptholemaei lochiensis Christus xylonicus, Parisiis, Ex officina Simonis Colinaei, 1531, $8^{\circ}$, Bibliothèque de l'Arsenal (RESERVE 8- BL- 5452).

50. Bartholamei Zamberti Deneti Comedia Dolotechne, (Impressum Venetiis per Ioannem de Tridino. librarium [...]), [après 1504], 4 ${ }^{\circ}$, [38] f., Shakespeare Folger Library (PA 8595 Z31 D5 1504 Cage).

51. Benoît Court, [Biographies de papes], [s.l.], [Xvi siècle], [9 f.], Bibliothèque municipale de Lyon (Rés Ms 195 T. 02).

52. I.e. « Moi, Benoît Court de Saint-Symphorien, chevalier de l'Église de Lyon, j'ai transcrit ce qui suit depuis un très ancien livre ».

53. In hoc volumine hec continentur, Platynae de vitis maxi. ponti. historia periocunda diligenter recognita et nunc tantum integre impressa, [...] Platynae contra amores dialogus ad Lodouicum stellam mantuanum, (Venetiis a Philippo pincio Mantuano 1511), $2^{\circ}, 168$-x f., Bibliothèque municipale de Lyon (Rés. Ms 195 T. 01).

54. Isaac Habert, Amours et Baisers, Nathalie Mahé (éd.), Genève, Droz, p. 283. Sur ce dialogue voir aussi Massimo Ciavolella, "Trois traités $\mathrm{du}_{\mathrm{Xv}} \mathrm{e}^{\mathrm{e}}$ siècle italien sur Anteros: Contra amores de Bartolomeo Sacchi, Anterotica de Pietro Edo et Anteros de Battista Fregoso » dans Ulrich Langer et Jan Miernowski (éd.), Anteros. Actes du colloque de Madison (Wisconsin), mars 1994, Orléans, Paradigme, 1994, p. 62-67.

55. In hoc volumine hec continentur, Platynae de vitis maxi. ponti. historia, op. cit., f. $\mathrm{C}^{1} \mathrm{v}$.

56. Sidonii apollinaris poema Aureum eiusdemque epistolae, (Impressum Mediolanni per magistrum Vldericum Scizenzeler. Impensis uenerabilium dominorum Presoyteri Hyeronimi 1497), $2^{\circ}$, [152] f., BmL (Rés. Inc 158 [1]).

57. Ibid., f. $\mathrm{d}^{4}$.

58. Ibid., f. $\mathrm{k}^{7} \mathrm{v}$.

\section{RÉSUMÉS}

En 1533, le juriste originaire des Monts du Lyonnais Benoît Court publie une édition commentée des Cinquante-et-un arrêts d'Amours de Martial d'Auvergne, chez Sébastien Gryphe, à Lyon. Les annotations latines intercalées dans le texte français des arrêts entendent expliquer des termes 
ou des sujets évoqués par Martial d'Auvergne. Les commentaires mettent alors en œuvre un dialogue entre de très nombreuses citations et références provenant des sommes du droit civil et canonique comme de sources extra-juridiques antiques, médiévales et modernes. Une très riche bibliothèque mentale se dégage ainsi de l'œuvre posant immédiatement la question de la provenance des sources de Court. Pour tenter d'apporter quelques éléments de réponse, cet article propose de confronter la bibliothèque mentale qui apparaît dans les commentaires avec les livres réels ayant appartenu au juriste.

In 1533, the jurist from the Monts du Lyonnais Benoît Court publishes a commented edition of Martial d'Auvergne's Cinquante-et-un arrêts d'Amours, at Sébastien Gryphe, in Lyon. The Latin annotations inserted in the French text of the judgments intend to explain terms or topics mentioned by Martial d'Auvergne. The comments then implement a dialogue between numerous quotations and references from sums of civil and canon laws as ancient, medieval and modern extra-legal sources. A very rich mental library thus emerges from the work immediately posing the question of the sources of Court. In order to bring some elements of answer, this article proposes to confront the mental library that appears in the comments with the real library which belonged to the jurist.

\section{INDEX}

Keywords : Court (Benoît), ancient library, reading practices, legal Humanism, erudition

Mots-clés : Court (Benoît), bibliothèque ancienne, pratiques de lecture, Humanisme juridique, érudition

\section{AUTEUR}

\section{HÉLÈNE LANNIER}

Université Lumière Lyon 2 - IHRIM (UMR 5317) /Centre Gabriel Naudé (EA 7286). 